\title{
Prognostic significance of neutrophil-to-lymphocyte ratio in cervical cancer: A systematic review and meta-analysis of observational studies
}

\author{
Qi-Tao Huang ${ }^{1}$, Qian-Qian Man², Jia Hu², Yi-Lin Yang ${ }^{2}$, Yue-Mei Zhang², Wei Wang ${ }^{1}$, \\ Mei Zhong ${ }^{1}$, Yan-Hong $\mathbf{Y u}^{1}$ \\ ${ }^{1}$ Division of Obstetrics and Gynecology, Nanfang Hospital, Southern Medical University, Guangzhou, 510515, China \\ ${ }^{2}$ Southern Medical University, Guangzhou, 510515, China \\ Correspondence to: Qi-Tao Huang, email: 15920454515@163.com \\ Mei Zhong, email: zhongm1960@163.com
}

Keywords: cervical cancer, inflammatory, neutrophil, lymphocyte, prognosis

Received: September 01, $2016 \quad$ Accepted: January 06, $2017 \quad$ Published: February 06, 2017

\section{ABSTRACT}

Background and aims: The prognostic role of neutrophil-to-lymphocyte ratio (NLR) in cervical cancer are controversial. We conducted this meta-analysis to obtain a more accurate assessment of prognostic significance of NLR in cervical cancer.

Results: A total of 9 studies, consisting of 2,804 patients, were selected in this meta-analysis. Our pooled results showed that high pre-treatment NLR level was significantly associated with poorer overall survival (HR: 1.88, 95\% CI 1.30-2.73) and shorter progression free survival (HR 1.65, 95\% CI 1.18-2.29). Additionally, increased NLR was also significantly correlated with tumor size (OR 2.05, 95\% CI 1.14-3.65), advanced FIGO stage (OR 2.12, 95\% CI1.28-3.49) and lymph node involvement (OR 2.24, 95\% CI 1.65-3.04).

Materials and Methods: We conducted a systematic literature search using the electronic databases PubMed, Web of Science, and Embase up to May 2016.Statistical analysis was performed using Stata 10.0.

Conclusions: Elevated pretreatment NLR could serve as a predicative factor of poor prognosis for cervical cancer patients.

\section{INTRODUCTION}

Cervical cancer is the second most common female malignancy, accounting for 500,000 new cases and over 200,000 deaths worldwide annually [1, 2]. Although tumor size, lymph node status, International Federation of Gynecology and Obstetrics (FIGO) staging criteria as well as pretreatment hemoglobin level were reported to be independent prognostic factors for cervical cancer [3-6], these factors are not routinely used in clinical settings due to insufficient specificity and sensitivity among patients. To further increase predictive accuracy, more prognostic parameters are still warranted.

Neutrophil to lymphocyte ratio (NLR), is a marker for evaluating the systemic potential balance between neutrophil-dependent pro-tumor inflammation and lymphocyte-associated anti-tumor immune response [7-8]. A higher level of NLR could represent a trend towards increased pro-tumor inflammation and decreased antitumor immune capacity.
Accumulating evidence demonstrates that NLR has prognostic significance in patients with various types of cancers [9-16]. Recently, several studies assessed the prognostic significance of NLR in patients with cervical cancer [17-25]. However, the prognostic significance of NLR in cervical cancer remained controversial. To clarify this issue, we performed this systematic review and metaanalysis to obtain a more reliable assessment of prognostic significance of NLR in patients with cervical cancer.

\section{RESULTS}

Selection and characteristics of included eligible studies

A flowchart for the selection of eligible studies is demonstrated in Figure 1. A total of 233 studies was retrieved and screened by title and abstract. 176 studies were excluded after the initial assessment of title and abstract. 
Among the remaining 57 articles, 36 were further excluded because they were letters, comments, editorials, or reviews. The full texts of the remaining 21 articles were evaluated. A total of 12 full-text articles were excluded, including 7 without available data and 5 without NLR category.

The basic information of the selected studies is summarized in Table 1.

\section{Association of pre-treatment NLR with overall survival}

The association between NLR and OS was assessed in 7 studies consisting of 2,804 patients. The pooled estimate indicated a significantly shorter OS in cervical cancer patients with high NLR compared to those with low NLR (HR: 1.88, 95\% CI 1.30-2.73) (Figure 2). Significant heterogeneity was observed among these studies $\left(\mathrm{I}^{2}=72.8 \%, P<0.001\right)$.

\section{Association of pre-treatment NLR with progression free survival}

The association between NLR and PFS was evaluated in 5 studies including 2,607 patients. Those with high pre-treatment NLR had a significantly poorer PFS than those with low NLR (HR 1.65, 95\% CI 1.18-2.29)
(Figure 3). Significant heterogeneity was observed among these studies $\left(\mathrm{I}^{2}=85.4 \%, P<0.001\right)$

\section{Correlations between NLR and clinical- pathological features}

The correlations between NLR and clinical features of cervical cancer are described below. NLR was positively related to tumor size (OR 2.05, 95\% CI 1.14-3.65) (Figure 4) and also demonstrated significant correlation with advanced FIGO stage (OR 2.12, 95\% CI 1.28-3.49) (Figure 5) with significant heterogeneity. Additionally, NLR was positively associated with lymph node involvement (OR 2.24, 95\% CI 1.65-3.04) (Figure 6) without significant heterogeneity.

\section{Subgroup analysis}

Results of subgroup meta-analyses are summarized in Table 2.

\section{Publication bias analysis}

The funnel plots showed a low probability of publication bias (Supplementary Figure 1 and 2). Consistently, the Egger's and Begger's regression tests

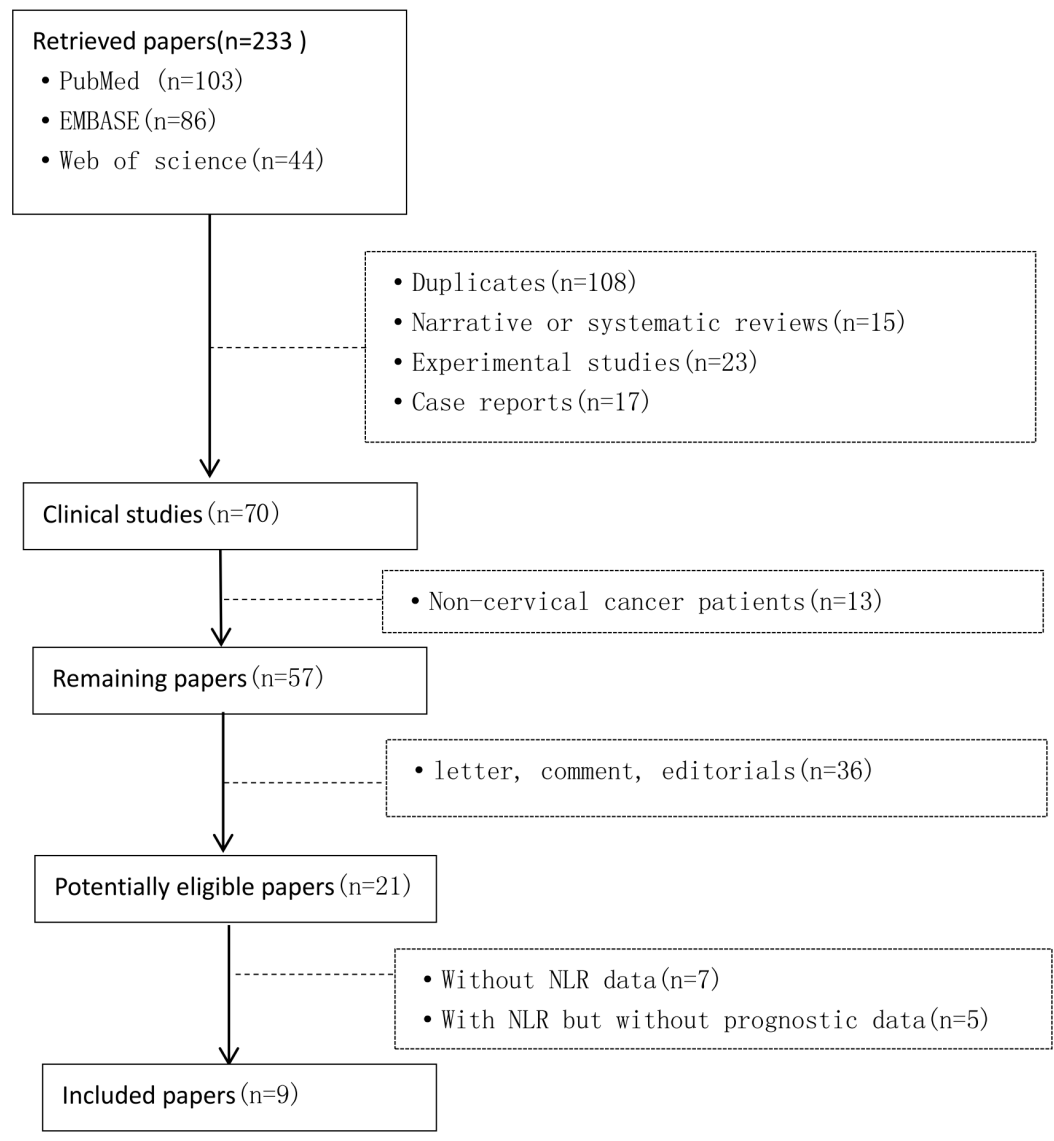

Figure 1: Flow diagram of the studies selection process. 
Table 1: Characteristics of all identified studies

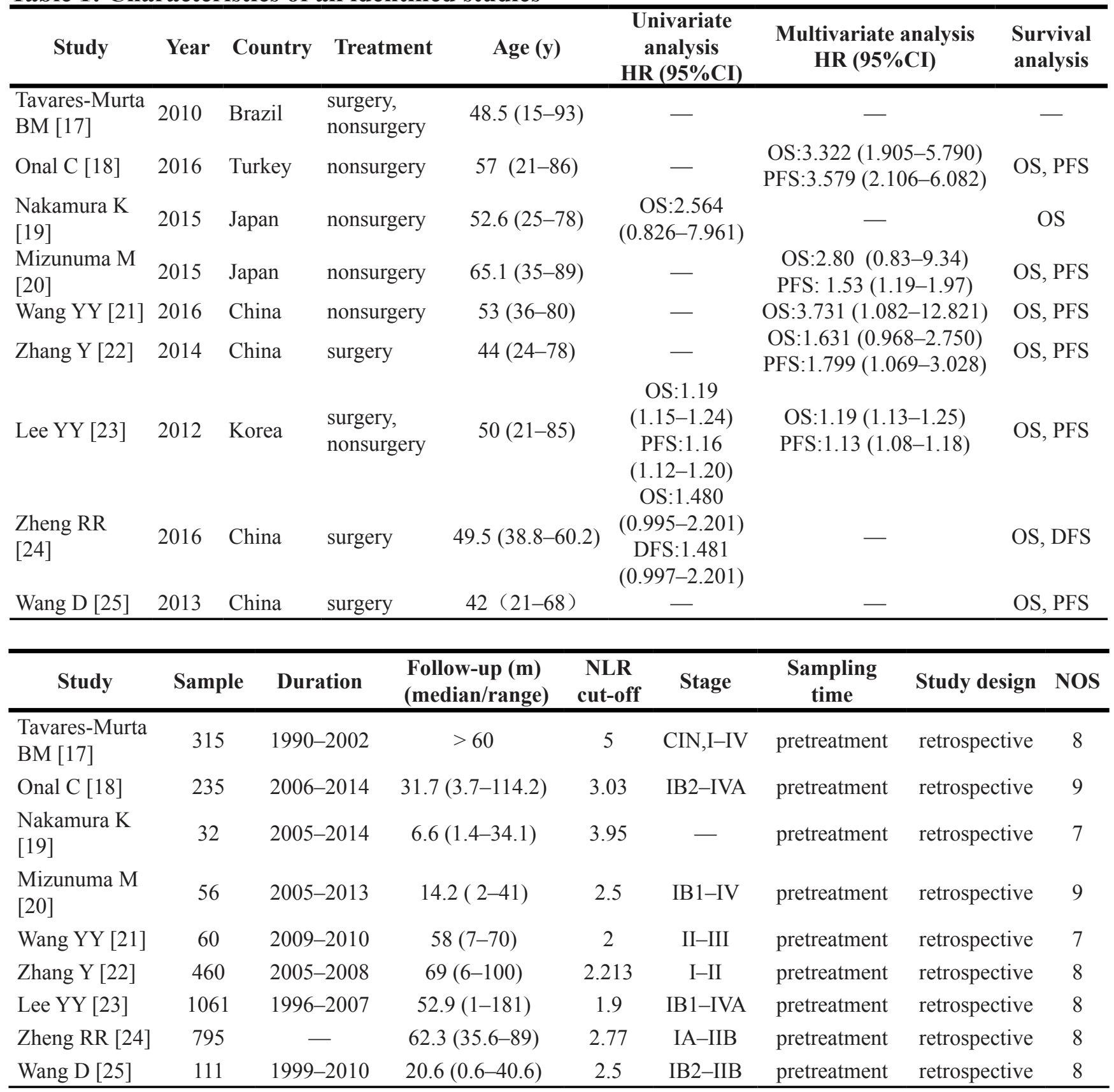

OS: overall survival; PFS: progression-free survival; DFS: disease-free survival;"_": not reported; Nonsurgery was defined as chemotherapy, radiotherapy or chemoradiotherapy.

demonstrated little evidence of publication bias for OS $(P=0.035 ; P=0.028)$ and for PFS $(P=0.221 ; P=0.033)$, respectively.

\section{Sensitivity analysis}

We performed a sensitivity analysis for every analysis by sequential omission of the individual study. The pooled HRs for OS and PFS were not significantly changed, which suggested the robustness of the results (Supplementary Figures 3 and 4).

\section{DISCUSSION}

Accumulating evidence demonstrated that inflammation exerted an essential role in cancer formation, development, and progression [26-30]. Neutrophils have been considered to be the primary source of circulating VEGF, which play a critical role in tumor-associated angiogenesis through producing many inflammatory cytokines such as tumor necrosis factor, interleukin 1, and providing a favorable micro-environment for tumor [28]. Conversely, lymphocytes exert a critical role in cancer- 
Table 2: Results of the meta-analysis on predictive value of NLR in cervical cancer.

\begin{tabular}{|c|c|c|c|c|c|c|c|c|}
\hline & \multicolumn{4}{|c|}{ Overall survival } & \multicolumn{4}{|c|}{ Progression free survival } \\
\hline & $\mathbf{N}$ & HR & LCI & HCI & $\mathbf{N}$ & HR & LCI & HCI \\
\hline Overall & 7 & 1.88 & 1.30 & 2.73 & 5 & 1.65 & 1.18 & 2.29 \\
\hline \multicolumn{9}{|l|}{ Geographic area } \\
\hline 1. Asian & 6 & 1.20 & 1.14 & 1.26 & 4 & 1.38 & 1.09 & 1.75 \\
\hline 2. non-Asian & 1 & 3.32 & 1.91 & 5.79 & 1 & 3.58 & 2.11 & 6.08 \\
\hline \multicolumn{9}{|l|}{ Statistical methods* } \\
\hline 1. Univariate & 3 & 1.19 & 1.15 & 1.24 & 2 & 1.16 & 1.12 & 1.20 \\
\hline 2. Multivariate & 5 & 2.05 & 1.20 & 3.50 & 4 & 1.71 & 1.14 & 2.56 \\
\hline \multicolumn{9}{|l|}{ Sample size } \\
\hline 1. $<300$ & 4 & 3.17 & 2.06 & 4.89 & 2 & 2.26 & 0.99 & 5.19 \\
\hline 2. $\geq 300$ & 3 & 1.20 & 1.14 & 1.26 & 3 & 1.32 & 1.00 & 1.75 \\
\hline \multicolumn{9}{|l|}{ Treatment } \\
\hline 1. Surgery & 2 & 1.53 & 1.12 & 2.10 & 2 & 1.59 & 1.16 & 2.18 \\
\hline 2. Chemoradiotherapy & 4 & 3.17 & 2.06 & 4.89 & 2 & 2.26 & 1.09 & 5.19 \\
\hline $\begin{array}{l}\text { 3. Surgery plus } \\
\text { chemoradiotherapy }\end{array}$ & 1 & 1.19 & 1.13 & 1.25 & 1 & 1.13 & 1.08 & 1.18 \\
\hline \multicolumn{9}{|l|}{ FIGO staging } \\
\hline 1. I-II & 2 & 1.53 & 1.12 & 2.10 & 2 & 1.59 & 1.16 & 2.18 \\
\hline 2. III-IV & 4 & 2.31 & 1.08 & 4.93 & 3 & 1.70 & 1.06 & 2.71 \\
\hline 3. I-IV & 1 & 2.56 & 0.83 & 7.96 & 0 & - & - & - \\
\hline \multicolumn{9}{|l|}{ NLR standard } \\
\hline 1. $<3$ & 5 & 1.20 & 1.14 & 1.26 & 4 & 1.38 & 1.09 & 1.75 \\
\hline $2 . \geq 3$ & 2 & 3.16 & 1.92 & 5.20 & 1 & 3.58 & 2.11 & 6.08 \\
\hline \multicolumn{9}{|l|}{ Follow-up } \\
\hline $1 .<18$ & 2 & 2.67 & 1.17 & 6.11 & 1 & 1.53 & 1.19 & 1.97 \\
\hline 2. $\geq 18$ & 5 & 1.77 & 1.19 & 2.65 & 4 & 1.73 & 1.08 & 2.78 \\
\hline
\end{tabular}

*The study (Lee YY 2012) reported HR (95\%CI) of both univariate and multivariate analysis results.

specific immune response [29]. It has been shown that an increased infiltration of lymphocytes into tumor tissue is associated with good prognosis [30]. In the present study, we found that an elevated NLR was associated with poorer OS and shorter PFS in cervical cancer patients, which was in accordance with the results from studies with several other cancer types.

Previous studies reported that higher pretreatment NLR had a stronger predictive effect in cancer at a more advanced stage [31, 32]. Yodying $\mathrm{H}$ et al. [31] evaluated the prognostic role of NLR in esophageal cancer and indicated that NLR was associated with tumor invasion and lymph node metastasis. Meanwhile, Xue TC et al. [32] found that NLR was associated with vascular invasion in hepatic carcinoma. In the present study, our results showed that
NLR was positively related to tumor size and significantly correlated with lymph node involvement as well as advanced tumor stage (FIGO). These observations indicated that inflammation severity might significantly affect intrinsic tumor characteristics in patients with cervical cancer.

Heterogeneity was observed in this meta-analysis. This heterogeneity may be partially caused by geographic area, statistical methods, sample size, NLR cut-off value, and the follow-up duration. In order to explore the source of heterogeneity in this meta-analysis, we performed a subgroup analysis. The subgroup analysis results demonstrated that the prognostic value of NLR was unaffected by the confounders mentioned above in the analysis. Moreover, the sensitivity analysis indicated that our results were relatively conclusive. 
There are several limitations that need to be addressed in this study. First, most of the studies selected in this meta-analysis were retrospective, observational studies, and no prospective cohort study was identified. Moreover, most studies only contained information of NLR predictive value in the same stages or in the same therapy. Therefore, they may be more susceptible to bias in data analysis and the predictive value of NLR in the same stages and the same therapy need to be explored in the future. Second, a previous systemic review demonstrated that increased NLR predicted poor PFS with prostate cancer only in Asians, but not in Caucasians, which could be attributed to the ethnicity heterogeneity [33]. In this meta-analysis, majority of included articles came from Asian countries. Therefore, our current conclusions may not be suitably applied to other populations. Third, only 9 studies were included and the cut-off value for defining high NLR in each individual study was different, ranging

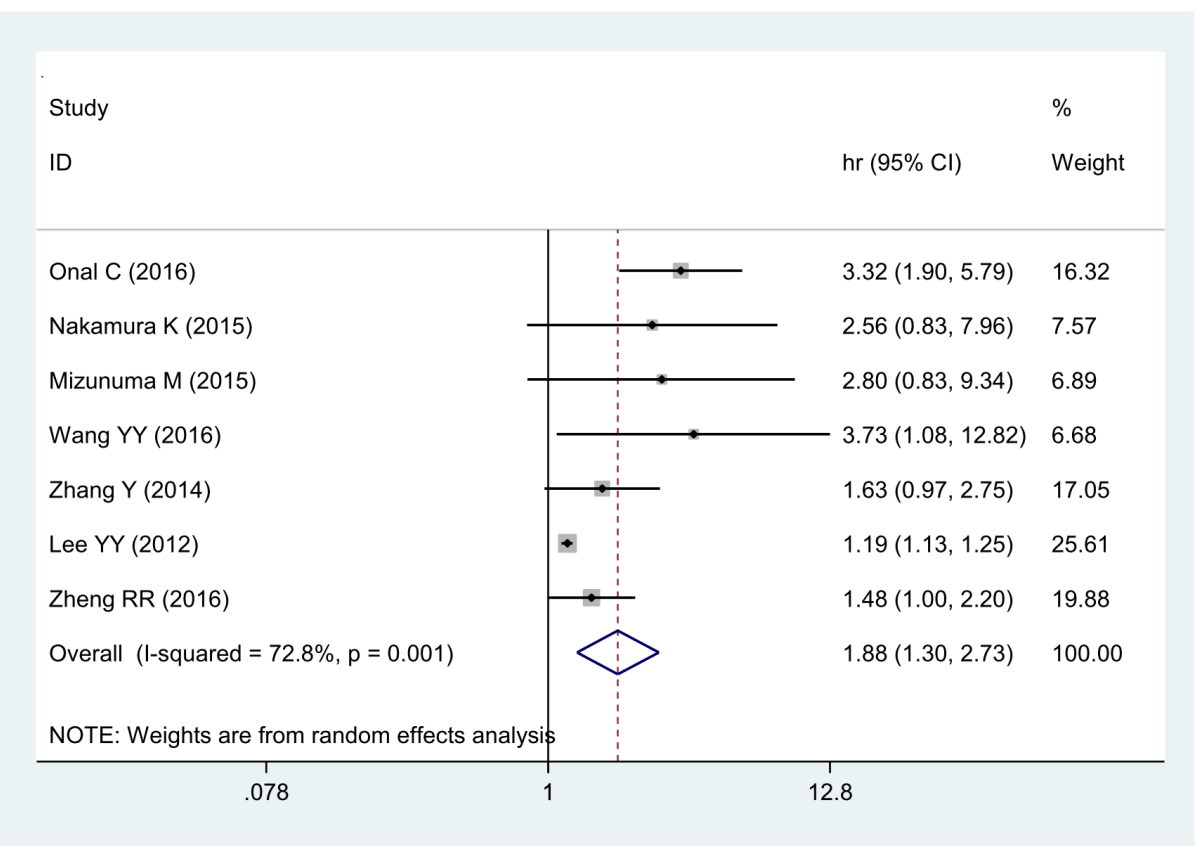

Figure 2: Forest plot of the correlation between NLR and OS in cervical cancer patients.

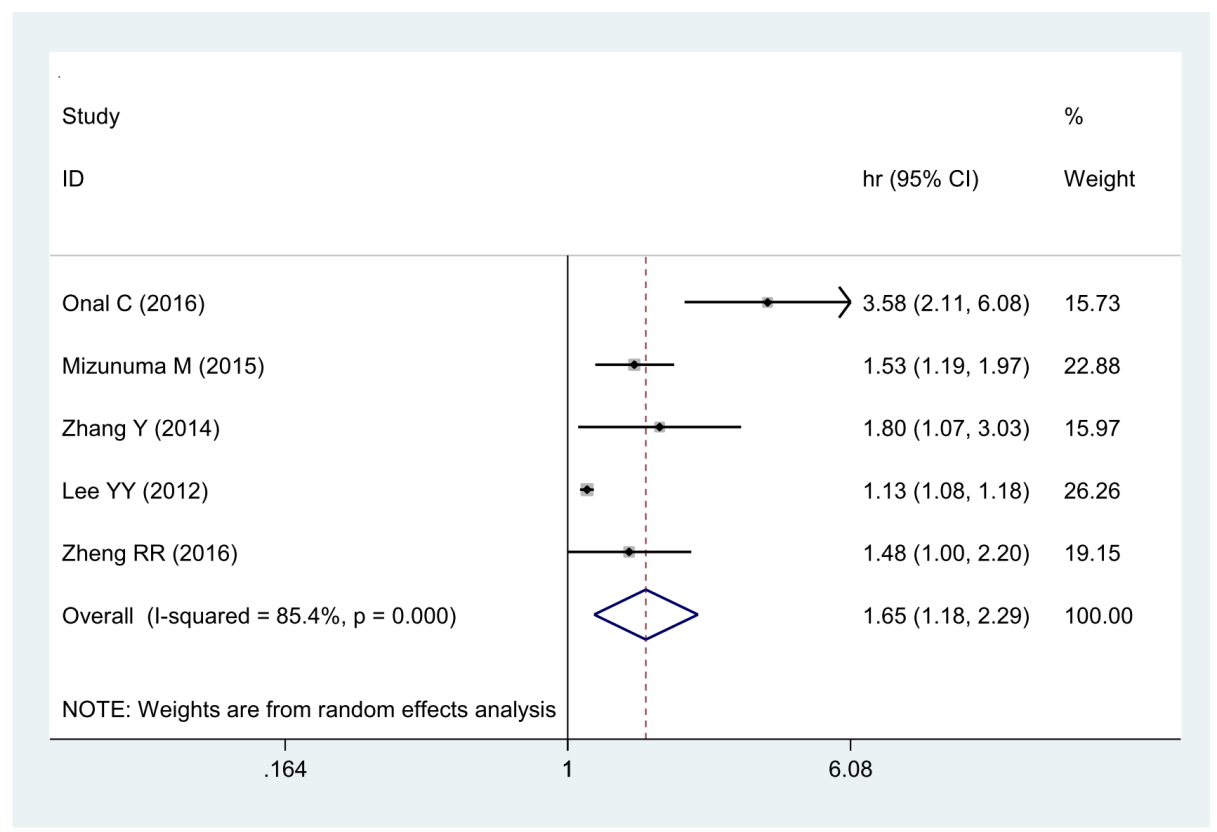

Figure 3: Forest plot of the correlation between NLR and PFS in cervical cancer patients. 
from 1.9 to 5 , which could hinder the application of this ratio in the clinical setting. In the future, original research is required to determine the most accurate cut-off value of NLR in cervical cancer patients.

Despite these limitations, our meta-analysis also has some strengths. To our knowledge, this meta-analysis is the first to evaluate the prognostic role of a pretreatment peripheral blood NLR in cervical cancer. Moreover, our results showed a significantly positive correlation between
NLR and the clinical features of cervical cancer, such as tumor size, FIGO stage, and lymph node involvement. Thus, NLR could have a wider clinical application regarding the prognostic assessment of cervical cancer, may be useful in stratifying patients, and in determining individual treatment plans in the future.

In conclusion, our meta-analysis of currently available clinical evidence demonstrated that NLR could serve as a promising prognostic marker of cervical cancer,

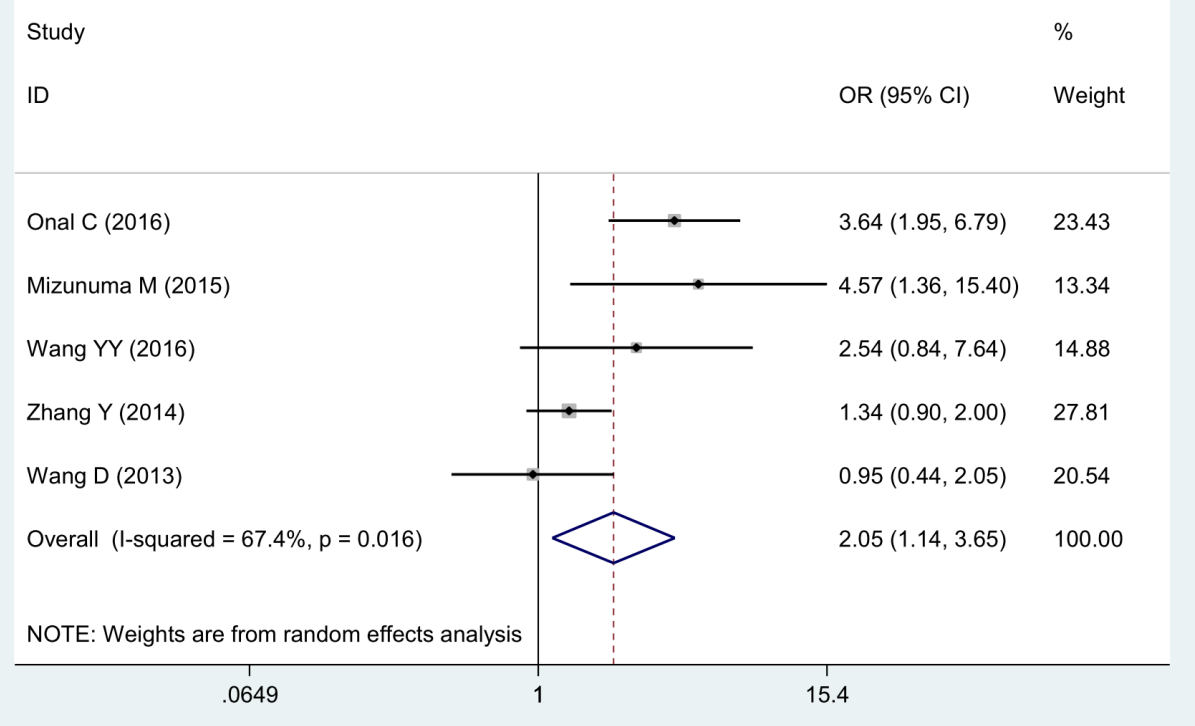

Figure 4: Forest plot of the correlation between NLR and tumor size in cervical cancer patients.

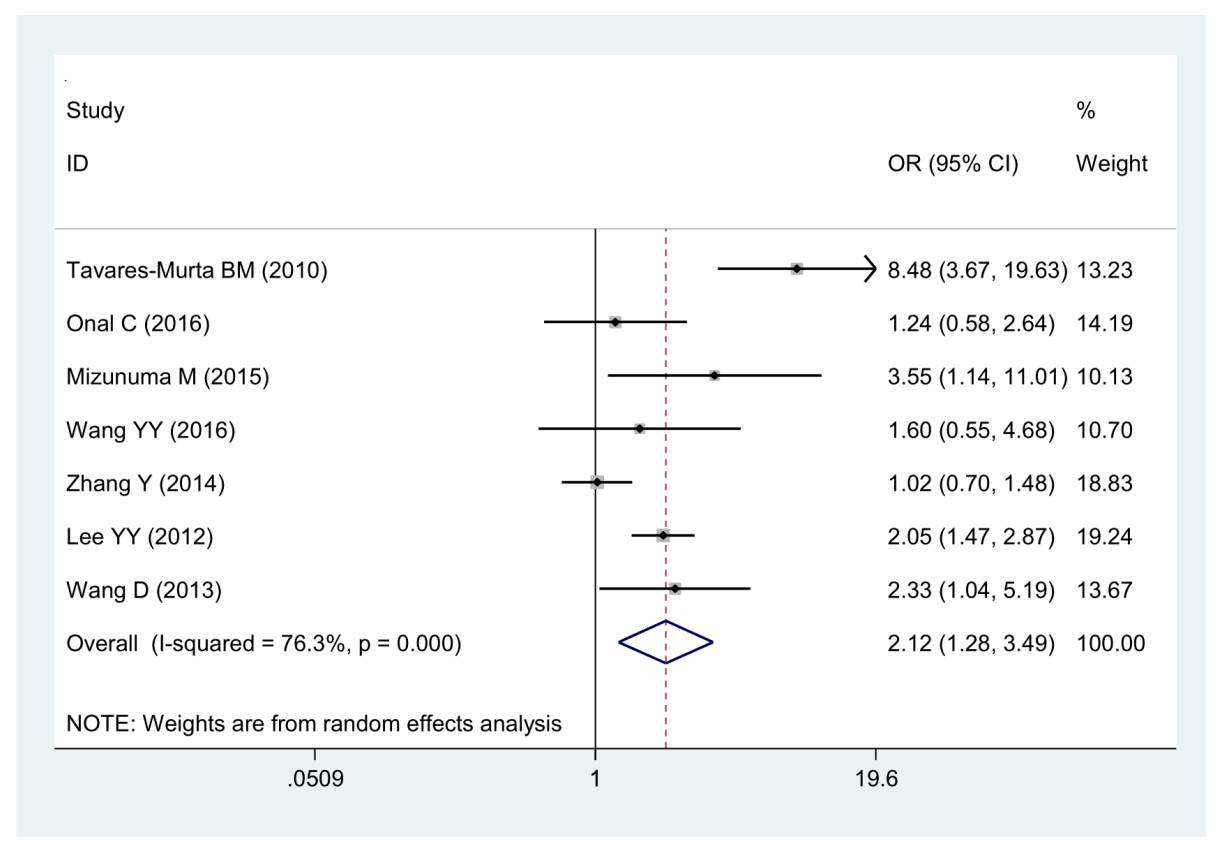

Figure 5: Forest plot of the correlation between NLR and FIGO staging in cervical cancer patients. 
because it is available from routine blood tests in daily clinical practice, which are convenient, low cost, and reproducible. In addition, considering that NLR level was associated with prognosis of cervical cancer, it would be interesting to explore whether decreasing the inflammatory conditions, such as lowing NLR level could serve as an adjuvant therapy and therefore prolong the survival of cervical cancer patients in the near future.

\section{MATERIALS AND METHODS}

This systematic review and meta-analysis was performed following the guidance provided in the Cochrane Handbook and was reported according to the Meta-analysis of Observational Studies in Epidemiology (MOOSE) guidelines [34].

\section{Search strategy}

We conducted a systematic literature search using the electronic databases PubMed, Web of Science, and Embase up to May 2016. Search terms included "neutrophil to lymphocyte ratio", "NLR", "cervix", "cervical" and "tumor, cancer, neoplasm, carcinoma or malignancy". The titles and abstracts of potential references were scanned carefully to exclude irrelevant articles. The remaining studies were assessed to identify the topic of interest, and full texts were then reviewed comprehensively.

\section{Selection criteria}

A study was included if it met the following criteria: (1) included patients with cervical cancer diagnosed histopathologically; (2) provided pre-treatment and/or post-treatment NLR and cut-off values, (3) evaluated the associations between pre-treatment and/or post-treatment NLR and survival outcomes. Exclusion criteria were (1) review articles, editorial comments, letters, expert opinion, conference abstracts, or case reports; (2) insufficient data for estimating hazard ratios (HRs) and 95\% confidence intervals (CIs); or (3) full text unavailable and non-English article.

All assessments were conducted independently by two reviewers to assure accuracy of inclusive studies. Multivariate data were preferred to univariate data if both were provided. However, univariate data were acceptable if no multivariate results were presented.

\section{Data extraction}

Two investigators independently gathered information from each eligible study. Data was extracted as follows: surname of first author, study country, year of publication, sample size, cancer stage, treatment method, cut-off value defining elevated NLR and HRs with 95\% CI for overall survival (OS) and progress-free survival (PFS)/recurrence-free survival (RFS). Disagreements in data extraction were resolved through discussion.

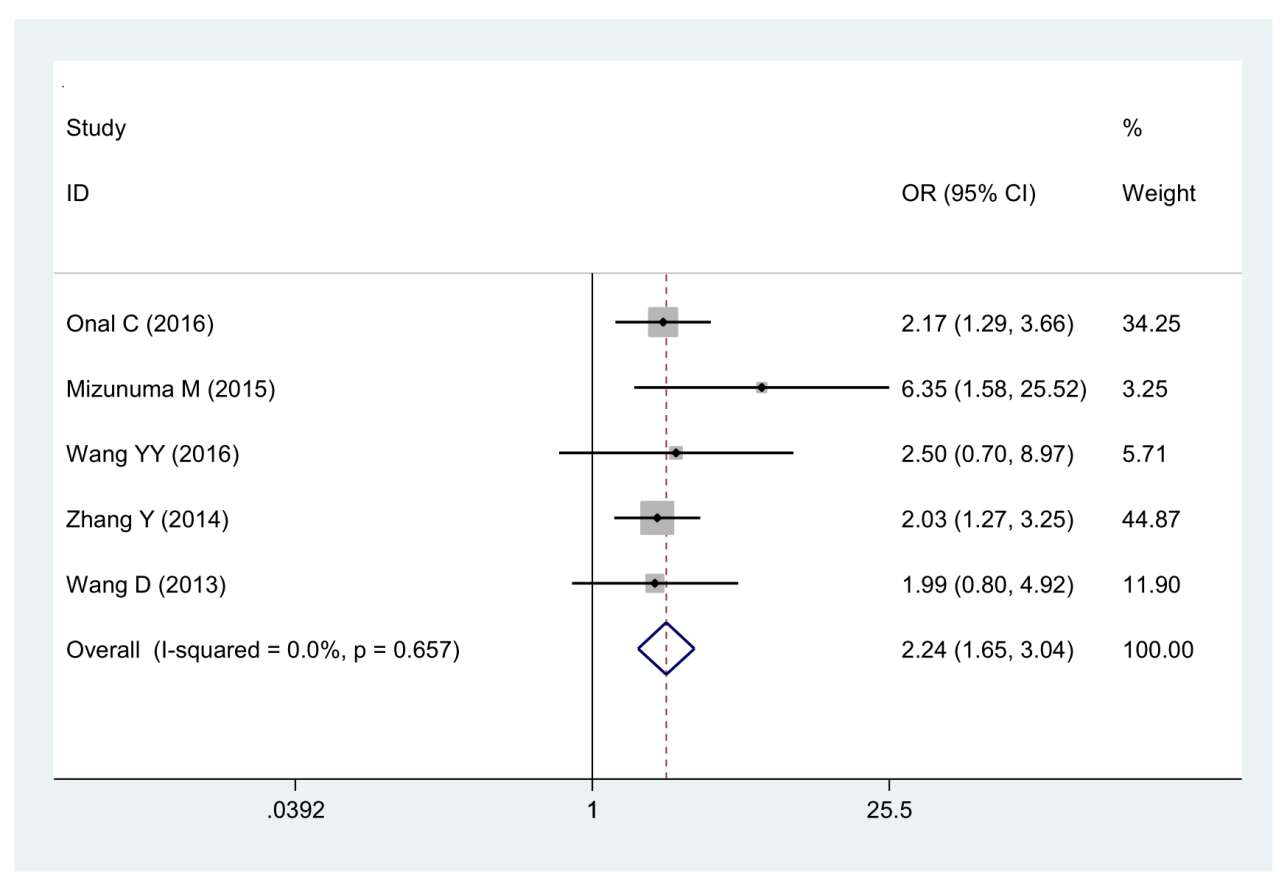

Figure 6: Forest plot of the correlation between NLR and lymph node metastasis in cervical cancer patients. 


\section{Assessment of methodological quality}

Two independent investigators assessed the quality of each included study using the Newcastle-Ottawa Quality Assessment Scale (NOS) [35]. On a score scale from 0 to 9 , a study with 7 or more stars was considered as high-quality.

\section{Statistical analysis}

The HR and corresponding 95\% CI were used to evaluate the prognostic efficiency of NLR on cervical cancer. In addition, the relationship between NLR and clinical-pathological features were reported as ORs and 95\% CIs. Cochran's $Q$ test and Higgins I-squared statistic were adopted to test the heterogeneity of pooled data. $I^{2}<50 \%$ and $p>0.1$ indicated no significant heterogeneity and fixed-effects model was applied to combine the effective value [36]. Otherwise, a random-effects model was adopted. All statistical analyses were performed using STATA 12.0 software (StataCorp LP, TX, USA).

Subgroup analyses were performed to investigate the associations of NLR with clinical features in relation to geographic area, statistical methods, sample size, cancer stage, lymph node involvement, NLR cut-off value, and follow-up duration. Moreover, a sensitivity analysis was performed to examine the robustness of the pooled results.

\section{CONFLICTS OF INTEREST}

The authors have no competing interests to declare.

\section{ETHICAL APPROVAL}

The study was approved by the local Institutional Review Board.

\section{FUNDING}

This work was supported by the National Natural Science Foundation of China (81401208, 81671466), Natural Science Foundation of Guangdong Province (2015A030310025) and the President Grant from Nanfang Hospital (2014B003).

\section{Authors'contributions}

Conceived and designed the experiments: QTH MZ YHY. Performed the experiments: QQM JH YMZ. Analyzed the data: QQM YLY. Contributed reagents/ materials/analysis tools: WW. Wrote the paper: QTH

\section{REFERENCES}

1. Gelband H, Sankaranarayanan R, Gauvreau CL, Horton S, Anderson BO, Bray F, Cleary J, Dare AJ, Denny L,
Gospodarowicz MK, Gupta S, Howard SC, Jaffray DA, et al. Costs, affordability, and feasibility of an essential package of cancer control interventions in low-income and middleincome countries: key messages from Disease Control Priorities, 3rd edition. Lancet. 2016; 387:2133-2144.

2. Forouzanfar MH, Foreman KJ, Delossantos AM, Lozano R, Lopez AD, Murray CJ, Naghavi M. Breast and cervical cancer in 187 countries between 1980 and 2010: a systematic analysis. Lancet. 2011; 378:1461-1484.

3. Ramirez PT, Pareja R, Rendon GJ, Millan C, Frumovitz M, Schmeler KM. Management of low-risk early-stage cervical cancer: should conization, simple trachelectomy, or simple hysterectomy replace radical surgery as the new standard of care? Gynecol Oncol. 2014; 132:254-259.

4. Intaraphet S, Kasatpibal N, Siriaunkgul S, Sogaard M, Patumanond J, Khunamornpong S, Chandacham A, Suprasert P. Prognostic impact of histology in patients with cervical squamous cell carcinoma, adenocarcinoma and small cell neuroendocrine carcinoma. Asian Pac J Cancer Prev. 2013; 14:5355-5360.

5. Demirci S, Ozsaran Z, Ozsaran A, Yavas F, Demircioglu B, Hanhan M, Dikmen Y, Aras AB. Evaluation of treatment results and prognostic factors in early-stage cervical carcinoma patients treated with postoperative radiotherapy or radiochemotherapy. Eur J Gynaecol Oncol. 2012; 33:62-67.

6. Horn LC, Bilek K, Fischer U, Einenkel J, Hentschel B. A cut-off value of $2 \mathrm{~cm}$ in tumor size is of prognostic value in surgically treated FIGO stage IB cervical cancer. Gynecol Oncol. 2014; 134:42-46.

7. Moses K, Brandau S. Human neutrophils: Their role in cancer and relation to myeloid-derived suppressor cells. Semin Immunol. 2016; 28:187-196.

8. Donskov F. Immunomonitoring and prognostic relevance of neutrophils in clinical trials. Semin Cancer Biol. 2013; 23:200-207.

9. Templeton AJ, McNamara MG, Seruga B, Vera-Badillo FE, Aneja P, Ocana A, Leibowitz-Amit R, Sonpavde G, Knox JJ, Tran B, Tannock IF, Amir E. Prognostic role of neutrophilto-lymphocyte ratio in solid tumors: a systematic review and meta-analysis. J Natl Cancer Inst. 2014; 106:u124.

10. Chen Y, Chen K, Xiao X, Nie Y, Qu S, Gong C, Su F, Song E. Pretreatment neutrophil-to-lymphocyte ratio is correlated with response to neoadjuvant chemotherapy as an independent prognostic indicator in breast cancer patients: a retrospective study. Bmc Cancer. 2016; 16:320.

11. Sun J, Chen X, Gao P, Song Y, Huang X, Yang Y, Zhao J, Ma B, Gao X, Wang Z. Can the Neutrophil to Lymphocyte Ratio Be Used to Determine Gastric Cancer Treatment Outcomes? A Systematic Review and Meta-Analysis. Dis Markers. 2016; 2016:7862469.

12. Malietzis G, Giacometti M, Kennedy RH, Athanasiou T, Aziz O, Jenkins JT. The emerging role of neutrophil to lymphocyte ratio in determining colorectal cancer treatment outcomes: a systematic review and meta-analysis. Ann Surg Oncol. 2014; 21:3938-3946. 
13. Szkandera J, Stotz M, Eisner F, Absenger G, Stojakovic T, Samonigg H, Kornprat P, Schaberl-Moser R, Alzoughbi W, Ress AL, Seggewies FS, Gerger A, Hoefler G, et al. External validation of the derived neutrophil to lymphocyte ratio as a prognostic marker on a large cohort of pancreatic cancer patients. Plos One. 2013; 8:e78225.

14. Lu SD, Wang YY, Peng NF, Peng YC, Zhong JH, Qin HG, Xiang BD, You XM, Ma L, Li LQ. Preoperative Ratio of Neutrophils to Lymphocytes Predicts PostresectionSurvival in Selected Patients With Early or Intermediate Stage Hepatocellular Carcinoma. Medicine (Baltimore). 2016; 95:e2722.

15. Berardi R, Rinaldi S, Santoni M, Newsom-Davis T, Tiberi M, Morgese F, Caramanti M, SaviniA, Ferrini C, Torniai M, Fiordoliva I, Bower M, Cascinu S. Prognostic models to predict survival in patients with advanced nonsmall cell lung cancer treated with first-line chemo- or targeted therapy. Oncotarget. 2016; 7:26916-26924. doi: 10.18632/oncotarget.8309.

16. Na N, Yao J, Cheng C, Huang Z, Hong L, Li H, Qiu J. Metaanalysis of the efficacy of the pretreatment neutrophil-tolymphocyte ratio as a predictor of prognosis in renal carcinoma patients receiving tyrosine kinase inhibitors. Oncotarget. 2016; 7:44039-44046. doi: 10.18632/oncotarget.9836.

17. Tavares-Murta BM, Mendonca MA, Duarte NL, Da SJ, Mutao TS, Garcia CB, Murta EF. Systemic leukocyte alterations are associated with invasive uterine cervical cancer. Int J Gynecol Cancer. 2010; 20:1154-1159.

18. Onal C, Guler OC, Yildirim BA. Prognostic Use of Pretreatment Hematologic Parameters in Patients Receiving Definitive Chemoradiotherapy for Cervical Cancer. Int J Gynecol Cancer. 2016; 26:1169-1175.

19. Nakamura K, Nishida T, Haruma T, Haraga J, Omichi C, Ogawa C, Kusumoto T, Seki N, Masuyama H, Hiramatsu Y. Pretreatment platelet-lymphocyte ratio is an independent predictor of cervical cancer recurrence following concurrent chemoradiation therapy. Mol Clin Oncol. 2015; 3:1001-1006.

20. Mizunuma M, Yokoyama Y, Futagami M, Aoki M, Takai Y, Mizunuma H. The pretreatment neutrophil-to-lymphocyte ratio predicts therapeutic response to radiation therapy and concurrent chemoradiation therapy in uterine cervical cancer. Int J Clin Oncol. 2015; 20:989-996.

21. Wang YY, Bai ZL, He JL, Yang Y, Zhao R, Hai P, Zhe H. Prognostic Value of Neutrophil-Related Factors in Locally Advanced Cervical Squamous Cell Carcinoma Patients Treated with Cisplatin-Based Concurrent Chemoradiotherapy. Dis Markers. 2016; 2016:3740794.

22. Zhang Y, Wang L, Liu Y, Wang S, Shang P, Gao Y, Chen X. Preoperative neutrophil-lymphocyte ratio before plateletlymphocyte ratio predicts clinical outcome in patients with cervical cancer treated with initial radical surgery. Int J Gynecol Cancer. 2014; 24:1319-1325.

23. Lee YY, Choi CH, Kim HJ, Kim TJ, Lee JW, Lee JH, Bae DS, Kim BG. Pretreatment neutrophil:lymphocyte ratio as a prognostic factor in cervical carcinoma. Anticancer Res. 2012; 32:1555-1561.

24. Zheng RR, Huang M, Jin C, Wang HC, Yu JT, Zeng LC, Zheng FY, Lin F. Cervical cancer systemic inflammation score: a novel predictor of prognosis. Oncotarget. 2016; 7:15230-15242. doi: 10.18632/oncotarget.7378.

25. Wang D, Wu M, Feng FZ, Huang HF, Yang JX, Shen K, Xiang Y. Pretreatment neutrophil-to-lymphocyte and platelet-to-lymphocyte ratios do not predict survival in patients with cervical cancer treated with neoadjuvant chemotherapy and radical hysterectomy. Chin Med J (Engl). 2013; 126:1464-1468.

26. Grivennikov SI, Greten FR, Karin M. Immunity, inflammation, and cancer. Cell. 2010; 140:883-899.

27. Mantovani A, Allavena P, Sica A, Balkwill F. Cancer-related inflammation. Nature. 2008; 454:436-444.

28. Mishalian I, Bayuh R, Levy L, Zolotarov L, Michaeli J, Fridlender ZG. Tumor-associated neutrophils (TAN) develop pro-tumorigenic properties during tumor progression. Cancer Immunol Immunother. 2013; 62:1745-1756.

29. Markl B, Wieberneit J, Kretsinger H, Mayr P, Anthuber M, Arnholdt HM, Schenkirsch G. Number of Intratumoral T Lymphocytes Is Associated With Lymph Node Size, Lymph Node Harvest, and Outcome in Node-Negative Colon Cancer. Am J Clin Pathol. 2016; 145:826-836.

30. Noble F, Mellows T, McCormick ML, Bateman AC, Harris S, Underwood TJ, Byrne JP, Bailey IS, Sharland DM, Kelly JJ, Primrose JN, Sahota SS, Bateman AR, Thomas GJ, Ottensmeier $\mathrm{CH}$. Tumour infiltrating lymphocytes correlate with improved survival in patients with oesophageal adenocarcinoma. Cancer Immunol Immunother. 2016; 65:651-662.

31. Yodying H, Matsuda A, Miyashita M, Matsumoto S, Sakurazawa N, Yamada M, Uchida E. Prognostic Significance of Neutrophil-to-Lymphocyte Ratio and Platelet-to-Lymphocyte Ratio in Oncologic Outcomes of Esophageal Cancer: A Systematic Review and Metaanalysis. Ann Surg Oncol. 2016; 23:646-654.

32. Xue TC, Jia QA, Ge NL, Chen Y, Zhang BH, Ye SL. Imbalance in systemic inflammation and immune response following transarterial chemoembolization potentially increases metastatic risk in huge hepatocellular carcinoma. Tumour Biol. 2015; 36:8797-8803.

33. Gu X, Gao X, Li X, Qi X, Ma M, Qin S, Yu H, Sun S, Zhou D, Wang W. Prognostic significance of neutrophil-tolymphocyte ratio in prostate cancer: evidence from 16,266 patients. Sci Rep. 2016; 6:22089.

34. Stroup DF, Berlin JA, Morton SC, Olkin I, Williamson GD, Rennie D, Moher D, Becker BJ, Sipe TA, Thacker SB. Metaanalysis of observational studies in epidemiology: a proposal for reporting. Meta-analysis Of Observational Studies in Epidemiology (MOOSE) group. JAMA. 2000; 283:2008-2012. 
35. Wells G, Shea BO, Connell D. The Newcastle-Ottawa Scale (Nos) For Assessing The Quality Of Nonrandomized Studies In Meta-Analysis. 2010; http://www.ohri.ca/ programs/clinical_epidemiology/oxford_web.ppt.
36. Higgins JP, Thompson SG, Deeks JJ, Altman DG. Measuring inconsistency in meta-analyses. BMJ. 2003; 327:557-560. 